\title{
LITHIUM ABUNDANCES AS A PROBE OF THE EARLY EVOLUTION OF SOLAR-TYPE STARS
}

\author{
Eduardo L. Martin \\ Institute d'Astrophysique de Paris, 98bis Bd Arago, F-75014 Paris \\ Instituto de Astrofisica de Canarias, E-38200 La Laguna,Tenerife, Spain \\ Gibor Basri \\ University of California at Berkeley, CA 94720, USA \\ Claude Bertout \\ Institute d'Astrophysique de Paris, 98bis Bd Arago, F-75014 Paris \\ Paper Presented by E. L. Martin
}

\section{Poster Paper}

Lithium is presently a rare element on the Sun. Nevertheless its abundance in the presolar nebula was two orders of magnitude higher than solar as inferred from meteorites (Nichiporuk and Moore, 1974 Geo. Cosmochim., Acta 38). The depletion of lithium on the Sun presumably started when the star was only a few million years old, central temperature was rising because of contraction, light elements like lithium were easily destroyed deep in the interior and rapid mixing with surface material was ensured by convection. We are interested in examining observationally the relation of lithium depletion and other characteristics of low-mass pre-main sequence evolution.

Associated with star-forming regions, there are many very young solar type stars $(M \leq 2 \mathrm{M} \odot$ and age $\leq 2 \times 10^{7} \mathrm{yr}$ ), generally known as T Tauri stars (TTS). In these stars lithium shows up as a strong photospheric line at $\lambda 6707 \AA$ and is taken as a spectral signature of youth (Herbig, 1962 Adv. Astr. Astroph.,1). Our aim is to compute lithium abundances on the basis of high quality data which is now becoming available. The most interesting points will be: (1) confronting observed lithium abundances to theoretical isochrones and evolutionary models (cf. Profitt and Michaud 1989, Ap.J., 346), (2) comparing populations of young clusters of different ages and comparing stars very similar in all properties but one (mass, age or rotational velocity), and (3) trying to differentiate the evolution of very young stars from the evolution of their circumstellar material.

Proper study of the lithium resonance line must take into account variations due to nonphotospheric continuum emission (possibly disk-star boundary layer) and thermal inhomogeneities similar to large sunspots and plages. Allowing for these effects leads to measurements of the true line strength of LiI $\lambda 6707$, which in turn can be used to estimate the photospheric $\mathrm{Li}$ abundance. The major drawback when passing from line strengths to chemical abundances comes from uncertainties in the atmospheric parameters like effective temperature, surface gravity, microturbulent velocity and metallicity appropriate for TTS. Thus, the study of lithium abundances encourages basic research on the properties of TTS atmospheres. Full discussion of our results will be presented elsewhere. 